\title{
Self-regulation strategies in blended learning environments in higher education: A systematic review
}

\author{
Jan Hein Eggers \\ Centre for Applied Research in Education, Amsterdam University of Applied Sciences, The Netherlands
}

\section{Ron Oostdam}

Centre for Applied Research in Education, Amsterdam University of Applied Sciences, The Netherlands; Research Institute of Child Development and Education, University of Amsterdam, The Netherlands

Joke Voogt

Research Institute of Child Development and Education, University of Amsterdam, The Netherlands

\begin{abstract}
Although self-regulation is an important feature related to students' study success as reflected in higher grades and less academic course delay, little is known about the role of selfregulation in blended learning environments in higher education. For this review, we analysed 21 studies in which self-regulation strategies were taught in the context of blended learning. Based on an analysis of literature, we identified four types of strategies: cognitive, metacognitive, motivational and management. Results show that most studies focused on metacognitive strategies, followed by cognitive strategies, whereas little to no attention is paid to motivation and management strategies. To facilitate self-regulation strategies nonhuman student tool interactional methods were most commonly used, followed by a mix of human student-teacher and non-human student content and student environment methods. Results further show that the extent to which students actively apply self-regulation strategies also depends heavily on teacher's actions within the blended learning environment. Measurement of self-regulation strategies is mainly done with questionnaires such as the Motivation and Self-regulation of Learning Questionnaire.
\end{abstract}

Implications for practice and policy:

- More attention to self-regulation in online and blended learning is essential.

- Lecturers and course designers of blended learning environments should be aware that four types of self-regulation strategies are important: cognitive, metacognitive, motivational and management.

- Within blended learning environments, more attention should be paid to cognitive, motivation and management strategies to promote self-regulation.

Keywords: blended learning, self-regulation, measurement instruments, intervention studies, systematic review

\section{Introduction}

A new trend in educational models emerged at the turn of the 20th century with blended learning (BL) as an approach to teaching and learning. Since then, BL has become a well-known concept in various forms of education (Spanjers et al., 2015) and is widely accepted in higher education (Allen et al., 2007). BL combines online learning and interaction opportunities with face-to-face teaching methods.

The definition of BL has long been confusing. Experts have given definitions emphasising various aspects (Driscoll, 2002). Definitions of BL generally cover three often-interrelated aspects of instruction and learning (Graham, 2006): first, BL as a mix of online and face-to face instruction (Ward \& LaBranche, 2003); second, as a mix of educational methods (Driscoll, 2002) and finally BL as a mix of educational modalities and/or delivery media (Bersin \& Associates, 2003). In this study, BL is considered as:

a formal educational program in which a student learns at least part through online delivery with some element of student control over time, place, path, and/or pace and at least in part at a supervised brick-and-mortar location away from home. (Staker \& Horn, 2012, p.3) 
BL in higher education offers many advantages over face-to-face learning. For example, students can study at their own pace (Harasim, 2000). This means, students are responsible for their own learning, can choose the desired online material, can communicate with the teacher and other students online and can attend classroom lessons (Dziuban et al., 2013). However, it appears that learning in BL environments places higher demands on the self-regulation of students (Bonk \& Graham, 2012). Learning in BL environments requires that students possess and can apply different self-regulated learning (SRL) strategies, such as setting goals and seeking help (Sharma et al., 2007).

Research shows that students' ability to use SRL strategies is positively associated with higher grades and less delay in course completion (cf. Pintrich, 2000). There are also indications that students who lack SRL strategies cannot learn successfully online (Azevedo et al., 2004). BL environments often do not adequately meet the needs of students who still lack sufficient SRL skills. Therefore, it is necessary to diagnose which support students need and which interactional methods are effective for developing SRL strategies in BL environments, and valid and reliable measurement instruments are required to evaluate students' proficiency level in SRL strategies (Barnard et al., 2009).

Interactional methods to facilitate SRL strategies in BL environments can be classified as human and nonhuman methods (Hanna et al., 2000). Human interactional methods can be divided into:

- $\quad$ student-teacher: dialogue between the student and the teacher, in which the teacher wants to arouse interest, clarify questions, guide and motivate

- $\quad$ student-student: dialogue between students, for example, an online discussion group

- $\quad$ student-experts: collaboration with experts or community members, for example, discussions of real-life situations with practitioners in the community.

Non-human interactional methods occur online and can be divided into:

- $\quad$ student tools: interaction in order to operate, manipulate and communicate with software, for example, online communication to share ideas

- student content: students' processing of the study material, for example, making sense of information available on the web

- $\quad$ student environment: sources and simulations provided by the environment, for example, webbased searches and online databases.

Reviews have investigated the implementation and effectiveness of educational interventions aimed at teaching and/or using SRL strategies (Broadbent \& Poon, 2015; de Bruijn-Smolders et al., 2016; Dignath et al., 2008; Hattie et al., 1996; Sitzmann \& Ely, 2011; Zheng, 2016). However, to our knowledge, no reviews have been conducted about the role of SRL strategies in BL environments in higher education. Although a recent review examined effective self-regulation processes in higher education (de BruijnSmolders et al., 2016), the authors did not specifically focus on SRL in BL environments. Other reviews (Broadbent \& Poon, 2015; Dignath et al., 2008; Hattie et al., 1996; Sitzmann \& Ely, 2011; Zheng, 2016) described SRL strategies in a holistic way, without specifying the underlying SRL strategies and interactional methods. Moreover, these reviews focused on academic outcomes and not on the promotion of self-regulation.

The main objective of this review is to contribute to our understanding of the role of SRL strategies in BL environments by answering the following research questions:

(1) Which interactional methods in BL environments are effective for teaching and/or using SRL strategies?

(2) Which instruments have been used to assess and measure SRL in BL contexts?

\section{Classification of SRL strategies}

SRL can be defined as an active, constructive process in which learners monitor, regulate and control their cognition, motivation and behaviour, guided and constrained by their goals and the features of the learning 
environment (Pintrich, 2000). Students who can regulate their own learning are considered as active, responsible learners acting purposefully to work towards their academic goals.

SRL is similar to self-directed learning (SDL), a central concept in adult learning, while SRL emerged from educational and cognitive psychology and is mostly utilised in the school environment. Both concepts are often used interchangeably (Dinsmore et al., 2008). They both relate to active and responsible learners who apply strategies to regulate, monitor and evaluate their learning process (Loyens et al., 2008). Unlike SRL, SDL states that the learner has autonomy in determining the content and approach to learning, regardless of educational standards (Loyens et al., 2008). SDL is a broader concept than SRL and implies that a learner is able to regulate, monitor and evaluate their own learning process. SRL is not by definition always identical with SDL (Loyens et al., 2008). Due to the fact that SDL implies SRL, studies on SDL are also included in this review.

SRL strategies can be classified in different ways (Boekaerts, 1997; Mayer, 2008). For this review, we applied the frequently used classification of Boekaerts (1997) with three main types of strategies: (a) cognitive strategies, such as elaboration, to deepen understanding of the studied domain, (b) metacognitive strategies, such as planning, to regulate the learning process and (c) motivational strategies, such as selfefficacy, to motivate oneself to learn. Considering the increasing number of new ways of accessing information and the amount of information accessible to students, Dignath et al. (2008) additionally defined a new category called management strategies, aimed at finding information on the Internet and constructing knowledge with peers. Given the potential relevance of these management strategies for BL environments, we added these to the classification of SRL strategies for this review (see Table 1).

Table 1

Classification of SRL strategies, divided into subcategories

\begin{tabular}{ll}
\hline Main strategies & Subcategories \\
\hline Cognitive strategies & Rehearsal \\
& Elaboration \\
Metacognitive strategies & Organisation \\
& Planning \\
Motivation strategies & Monitoring \\
& Evaluation \\
Management strategies & Self-efficacy \\
& Task value \\
& Goal orientation \\
& Effort \\
\hline
\end{tabular}

Cognitive strategies help students to influence the process of receiving information, encoding information in memory and retrieving it when needed (Duncan \& McKeachie, 2005). Cognitive strategies are divided into three subcategories (Pintrich et al., 1991). Rehearsal strategies focus on repeating material in order to facilitate learning. Elaboration strategies help students to store information into their long-term memory by building internal connections between the items to be learned. Examples are summarising or expressing ideas in one's own words. Finally, organisation strategies support the process of selecting information, making connections between different elements or creating meaningful units of information, for example, text structuring and information categorisation (Weinstein et al., 2000).

Metacognitive strategies refer to acquired knowledge about cognitive approaches, knowledge that can be applied for mentally managing the use of strategies. These strategies are divided in planning, monitoring and evaluation (Schraw \& Dennison, 1994). Planning strategies are deployed for planning learning in an efficient way. Examples are creating a plan for the deliverables and estimating how much time to spend on them. Monitoring strategies are used to check progress in learning and to adjust the approach accordingly. Examples are checking understanding and validating information obtained. Evaluation strategies can be used to analyse task performance and/or the effectiveness of the approach applied, for example, reviewing a text and comparing the outcome to the goal (Boekaerts, 1997).

Motivation strategies are aimed at increasing specific types of stimuli, whether to participate in or refrain from strategy use. Motivation strategies are divided into self-efficacy, task value and goal orientation. Self- 
efficacy refers to students' belief in their ability to complete a task and confidence in the skills needed to perform it (Pintrich, 2003). Task value refers to beliefs about how valuable and meaningful an academic task is. Lastly, goal orientation refers to the reasons why students perform a task, which can be intrinsic factors such as curiosity, control of skills or extrinsic factors like competition (Harackiewicz et al., 2002).

Management strategies are applied to achieve optimal learning circumstances and are divided into three subcategories: effort, environmental and peer. The strategies are aimed at helping students achieve their learning goals in case of difficulties or distractions (Dignath et al., 2008), for example, when a student persists in study, even if the teaching material is uninteresting. Environmental management strategies can be applied for enhancing or optimising the learning environment, for example, finding a quiet place to study or using the Internet to search for information. Peer management relates to knowledge about collaboration, for example, requesting fellow students for help, using efficient forms of cooperation (Pintrich et al., 1991).

\section{Method}

\section{Search design}

We conducted an extensive literature review to identify and retrieve empirical studies relevant to answering the research questions. We formulated the following search string, with some variations to take into account for specific retrieval sources: ("blended learn*" OR "blended instruction*" OR "blended teach*" OR "hybrid learning" OR "hybrid instruction*" OR "hybrid teaching" OR "hybrid course*" OR "flipped classroom*" OR "flipped learn*") AND ("self-regulat*" OR selfregulat* OR SRL OR "self-direct*" OR "self-manag*" OR "meta-cognit*" OR metacognit*) AND (college* OR undergrad* OR "tertiary education" OR "tertiary school*" OR postsecondary OR "post-secondary" OR universit* OR "higher education" OR student* OR freshm*n OR sophomore*). We searched the following electronic databases and engines: ERIC, PsycINFO, Web of Science, Scopus and Google Scholar. We used Google Scholar to search for additional sources, but an inspection of the first 200 sources did not yield new publications. The search was limited to peer-reviewed studies in English published from 2000 onwards due to the first use of the term BL in that year (Bliuc et al., 2007). We conducted the search in March 2019.

\section{Selection process and criteria}

The search resulted in 691 records (see Figure 1). Duplicates $(n=312)$ were identified and removed. Full reports or summaries were obtained for each title that seemed to meet the inclusion criteria (see Table 2).

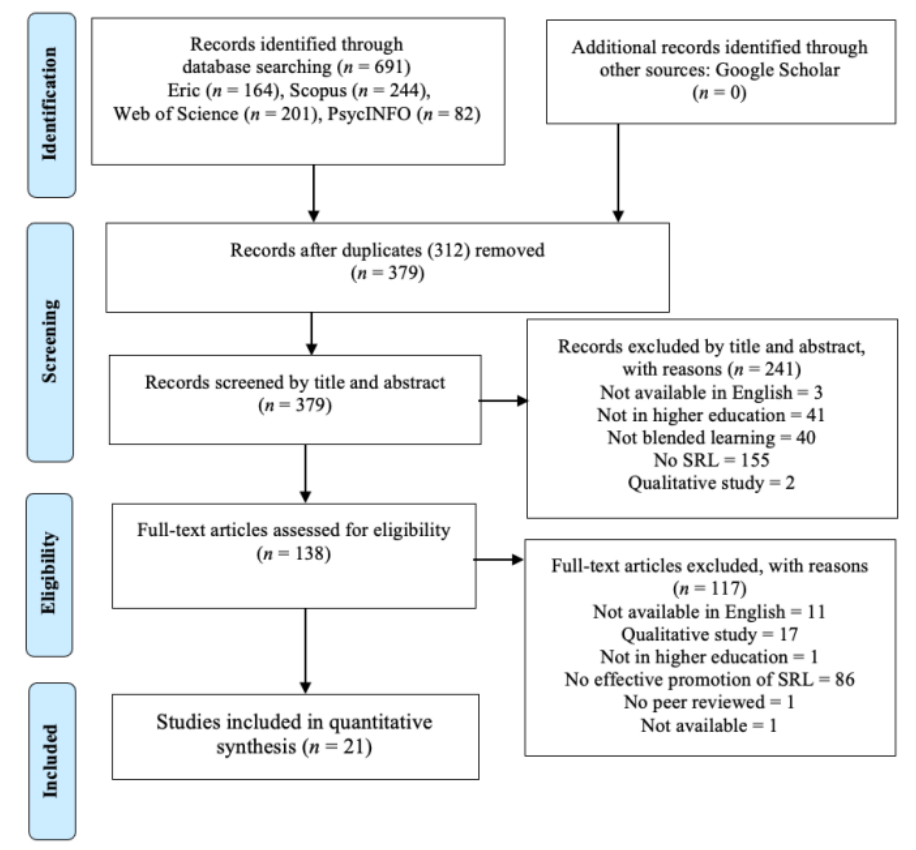

Figure 1. Flow diagram of selection of papers (adapted from Moher et al., 2009, p.267) 
The first author independently screened titles and abstracts of the remaining manuscripts $(n=379)$ and subsequently examined full texts to determine whether an article was eligible for inclusion. Any doubt about the inclusion was discussed with the other authors until consensus was reached. In total, 241 articles were excluded in view of their titles and abstracts. Furthermore, 117 full-text articles were excluded for two reasons: (a) the study was not directed to the acquisition or promoting of SRL strategies and (b) it was not a quantitative study. Most studies that were excluded focused only on achieving higher grades, did not examine whether SLR strategies were taught or did not explain how SRL strategies was measured.

Table 2

Inclusion criteria

\begin{tabular}{ll}
\hline Criteria & Inclusion \\
\hline Target audience & Higher education \\
Learning environment & BL \\
Studies & Empirical intervention studies focused upon acquiring and promoting SRL \\
& strategies and/or measuring SRL strategies \\
Articles & Peer-reviewed scholarly journals \\
Period & 2000 onwards \\
Language & English \\
\hline
\end{tabular}

\section{Data analysis}

We developed a coding scheme to systematically analyse the retrieved studies. We tested the scheme and refined it until we agreed on the topics and the corresponding categories. For answering the first research question, we first coded which SRL strategies were addressed in the intervention. Secondly, we coded each study whether the interactional method was human, non-human or a combination of both (Hanna et al., 2000). For answering the second research question, we coded characteristics of the measurement instruments for SRL strategies: name, format (questionnaire, self-report, interview, test with items), type of SRL strategy, psychometric quality (reliability indices and validity). Furthermore, we classified the intervention effects on SRL strategies as reported by the authors, preferably in terms of effect sizes (Cohen's $d$ ).

\section{Results}

\section{General study characteristics}

Remarkably, none of the studies were conducted before 2013 and most of them were published in 2018. An overview of the general characteristics of the studies is presented in Table 3. Between 32 and 1,027 participants were involved in the various studies. The majority of the studies had an audience size up to 150 participants $(n=15)$. Most studies took place within social science $(n=12)$ or science $(n=5)$ programs. Four studies had not specified the educational context. Participants were mainly undergraduate students. Most studies applied a quasi-experimental pre-posttest design with an experimental treatment and control group $(n=12)$. Experimental studies with pre-posttest $(n=2)$ or posttest only design $(n=1)$ were rare. Additionally, there were relatively many studies with a one group only pre-posttest $(n=3)$ or posttest only design $(n=3)$.

Most studies used the flipped classroom (FC) $(n=14)$ followed by studies with BL $(n=7)$ as a treatment group. In some experiments, a FC $(n=7)$, followed with traditional teaching $(n=5)$ and BL $(n=2)$, was used as a control group. 
Table 3

General characteristics of the studies, by author(s), country, aim of the intervention, participants, cluster, course, research design, core features of treatment and control groups

\begin{tabular}{|c|c|c|c|c|c|c|c|c|c|}
\hline No. & Author(s) & Ctry & $\begin{array}{l}\text { Aim of the } \\
\text { intervention }\end{array}$ & Participants & Cluster & Course & $\begin{array}{l}\text { Research } \\
\text { design }\end{array}$ & $\begin{array}{l}\text { Core } \\
\text { features } \\
\text { treatment }\end{array}$ & $\begin{array}{l}\text { Control } \\
\text { groups }\end{array}$ \\
\hline 1 & $\begin{array}{l}\text { Alsancak } \\
\text { Sirakaya \& } \\
\text { Ozdemir } \\
(2018)\end{array}$ & TR & $\begin{array}{l}\text { Examine effects } \\
\text { of FC model on } \\
\text { SDL skills \& } \\
\text { motivation }\end{array}$ & $\begin{array}{l}\text { US } \\
(n=66)\end{array}$ & SS & RESM & QE-PP & $\begin{array}{l}\mathrm{FC} \\
(n=32)\end{array}$ & $\begin{array}{l}\mathrm{BL} \\
(n=34)\end{array}$ \\
\hline 2 & $\begin{array}{l}\text { Atwa et al. } \\
\text { (2018) }\end{array}$ & $\mathrm{AU}$ & $\begin{array}{l}\text { Examine design } \\
\text { of novel hybrid } \\
\text { pedagogy using } \\
\text { team-based \& } \\
\text { case-based } \\
\text { learning to } \\
\text { promote self- } \\
\text { regulation, } \\
\text { engagement \& } \\
\text { motivation of } \\
\text { students with low } \\
\text { AU tertiary } \\
\text { admission ranks } \\
\text { at entry to } \\
\text { university }\end{array}$ & $\begin{array}{l}\text { 1st year } \\
(n=314)\end{array}$ & S & BDYS & QE-PP & $\begin{array}{l}\text { BL \& TB } \\
\& \text { CBL } \\
(n=75)\end{array}$ & $\begin{array}{l}1 . \mathrm{TT} \\
(n=75) \\
2 . \mathrm{TT} \& \\
\text { no } \\
\text { scaffold } \\
\text { clinic } \\
\text { cases \& } \\
\text { CBL } \\
(n= \\
164)\end{array}$ \\
\hline 3 & $\begin{array}{l}\text { Chen \& } \\
\text { Hwang (2018) }\end{array}$ & TW & $\begin{array}{l}\text { Examine effects } \\
\text { of integrated FC } \\
\& \text { IRS-facilitated } \\
\text { collective issue- } \\
\text { quest approach } \\
\text { on learning } \\
\text { performance, } \\
\text { self-regulation, } \\
\text { collective } \\
\text { efficacy \& } \\
\text { satisfaction }\end{array}$ & $\begin{array}{l}\text { UG } \\
(n=85)\end{array}$ & NM & MKT & QE-PP & $\begin{array}{l}\text { FC \& } \\
\text { IRS- } \\
\text { collective } \\
\text { issue- } \\
\text { quest } \\
\text { strategy } \\
(n=42)\end{array}$ & $\begin{array}{l}\text { FC only } \\
(n=43)\end{array}$ \\
\hline 4 & $\begin{array}{l}\text { Chyr et al. } \\
\text { (2017) }\end{array}$ & TW & $\begin{array}{l}\text { Explore effects } \\
\text { of online } \\
\text { academic help- } \\
\text { seeking \& FL on } \\
\text { improving } \\
\text { students' } \\
\text { learning }\end{array}$ & $\begin{array}{l}\text { 1st year } \\
(n=102)\end{array}$ & SS & OS & QE-PP & $\begin{array}{l}\text { 1. FC \& } \\
\text { online } \\
\text { help- } \\
\text { seeking } \\
(n=33) \\
2 . \text { FC } \\
(n=34)\end{array}$ & $\begin{array}{l}\mathrm{TT} \\
(n=35)\end{array}$ \\
\hline 5 & $\begin{array}{l}\text { El-Senousy \& } \\
\text { Alquda (2017) }\end{array}$ & SA & $\begin{array}{l}\text { Explore effects } \\
\text { of FC strategy } \\
\text { using Blackboard } \\
\text { mash-up tools in } \\
\text { enhancing } \\
\text { achievement \& } \\
\text { SRL skills of } \\
\text { university } \\
\text { students }\end{array}$ & $\begin{array}{l}\text { US } \\
(n=60)\end{array}$ & S & $\begin{array}{l}\text { CMP } \\
101 N\end{array}$ & E-PP & $\begin{array}{l}\text { FC \& } \\
\text { Blackboar } \\
\text { d mash-up } \\
\text { sources } \\
(n=30)\end{array}$ & $\begin{array}{l}\text { TT } \\
(n=30)\end{array}$ \\
\hline 6 & $\begin{array}{l}\text { Ferrer- } \\
\text { Torregros et } \\
\text { al. (2016) }\end{array}$ & ES & $\begin{array}{l}\text { Examine didactic } \\
\text { strategies \& } \\
\text { associated DL } \\
\text { aids based on FC } \\
\text { where } \\
\text { transmitting } \\
\text { information is } \\
\text { via teacher- } \\
\text { prepared aids, } \\
\text { ensuring students } \\
\text { work } \\
\text { independently } \\
\text { before class }\end{array}$ & $\begin{array}{l}\text { 1st year } \\
(n=171)\end{array}$ & SS & MOTF & E-P & $\begin{array}{l}\text { 1. FC \& } \\
\text { instructor } \\
\text { notes \& } \\
\text { videos } \\
(n=51) \\
2 . \text { FC \& } \\
\text { instructor } \\
\text { notes \& } \\
\text { AR } \\
(n=60)\end{array}$ & $\begin{array}{l}\text { FC \& } \\
\text { instructo } \\
\text { r notes } \\
(n=60)\end{array}$ \\
\hline 7 & $\begin{array}{l}\text { Giacumo \& } \\
\text { Savenye } \\
(2019)\end{array}$ & USA & $\begin{array}{l}\text { Test effects of } \\
\text { two } \\
\text { metacognitive } \\
\text { scaffolds on } \\
\text { cognition by } \\
\text { evaluating } \\
\text { student critical } \\
\text { skill performance } \\
\text { in asynchronous } \\
\text { discussion board }\end{array}$ & $\begin{array}{l}\mathrm{UG} \\
(n=257)\end{array}$ & SS & LEUP & QE-PP & $\begin{array}{l}\text { 1. BL + } \\
\text { instructor } \\
\text { prompts } \\
2 . \mathrm{BL} \& \\
\text { rubric } \\
\text { 3. BL } \\
\text { instructor } \\
\text { prompts } \\
\text { \& rubric }\end{array}$ & $\begin{array}{l}\text { BL no } \\
\text { rubric \& } \\
\text { no } \\
\text { prompts }\end{array}$ \\
\hline
\end{tabular}




\begin{tabular}{|c|c|c|c|c|c|c|c|c|c|}
\hline No. & Author(s) & Ctry & $\begin{array}{l}\text { Aim of the } \\
\text { intervention }\end{array}$ & Participants & Cluster & Course & $\begin{array}{l}\text { Research } \\
\text { design }\end{array}$ & $\begin{array}{l}\text { Core } \\
\text { features } \\
\text { treatment }\end{array}$ & $\begin{array}{l}\text { Control } \\
\text { groups }\end{array}$ \\
\hline 8 & $\begin{array}{l}\text { Hsu \& Hsieh } \\
(2014)\end{array}$ & $\mathrm{TW}$ & $\begin{array}{l}\& \text { achievement } \\
\text { in a BL module } \\
\text { Examine } \\
\text { influence of } \\
\text { demographic, } \\
\text { learning } \\
\text { involvement \& } \\
\text { learning } \\
\text { performance } \\
\text { variables on } \\
\text { metacognition of } \\
\text { UG nursing } \\
\text { students in a BL } \\
\text { environment }\end{array}$ & $\begin{array}{l}\mathrm{UG} \\
(n=99)\end{array}$ & SS & $\mathrm{NE}$ & OG-P & $\begin{array}{l}\text { BL (2 } \\
\text { classes), } \\
\text { online } \\
\text { video \& } \\
\text { exchange } \\
\text { through } \\
\text { online } \\
\text { chat room }\end{array}$ & - \\
\hline 9 & $\begin{array}{l}\text { Karaoğlan } \\
\text { Y1lmaz et al. } \\
(2018)\end{array}$ & TR & $\begin{array}{l}\text { Examine effects } \\
\text { of metacognitive } \\
\text { support via } \\
\text { pedagogical } \\
\text { agent on } \\
\text { students' self- } \\
\text { regulation skills }\end{array}$ & $\begin{array}{l}1 \text { st year } \\
(n=102)\end{array}$ & $\mathrm{NM}$ & CMP-I & QE-PP & $\begin{array}{l}\text { FC \& PA } \\
\& \text { MS } \\
\text { platform } \\
(n=52)\end{array}$ & $\begin{array}{l}\mathrm{FC} \\
(n=50)\end{array}$ \\
\hline 10 & $\begin{array}{l}\text { Kassab et al. } \\
(2015)\end{array}$ & IE & $\begin{array}{l}\text { Examine the } \\
\text { relationship } \\
\text { between aspects } \\
\text { of course } \\
\text { experience, SRL, } \\
\text { \& academic } \\
\text { achievement of } \\
\text { medical students } \\
\text { in a BL } \\
\text { curriculum }\end{array}$ & $\begin{array}{l}2 \text { nd year } \\
(n=171)\end{array}$ & SS & $\mathrm{JCM}$ & OG-P & $\begin{array}{l}\text { BL \& } \\
\text { virtual } \\
\text { learning } \\
(n=171)\end{array}$ & - \\
\hline 11 & $\begin{array}{l}\text { Ma et al. } \\
\text { (2018) }\end{array}$ & $\mathrm{CN}$ & $\begin{array}{l}\text { Assess students' } \\
\text { perceptions of } \\
\text { application-based } \\
\text { FC teaching } \\
\text { model in an } \\
\text { immunology } \\
\text { course }\end{array}$ & $\begin{array}{l}\text { 2nd year } \\
(n=92)\end{array}$ & SS & MI & QE-PP & $\begin{array}{l}\text { APP-FC } \\
\& \text { mobile } \\
\text { phone app } \\
(n=42)\end{array}$ & $\begin{array}{l}\mathrm{TT} \\
(n=50)\end{array}$ \\
\hline 12 & $\begin{array}{l}\text { Moos \& } \\
\text { Bonde (2016) }\end{array}$ & USA & $\begin{array}{l}\text { Examine } \\
\text { effectiveness of } \\
\text { embedding SRL } \\
\text { prompts in a } \\
\text { video designed } \\
\text { for FC model }\end{array}$ & $\begin{array}{l}\text { UG } \\
(n=32)\end{array}$ & SS & PSY & QE-PP & $\begin{array}{l}\text { FC \& } \\
\text { video \& } \\
\text { SRL } \\
\text { prompts } \\
(n=16)\end{array}$ & $\begin{array}{l}\mathrm{FC} \mathrm{\&} \\
\text { video } \\
(n=16)\end{array}$ \\
\hline 13 & Ng (2018) & $\mathrm{CN}$ & $\begin{array}{l}\text { Examine whether } \\
\text { FC with } \\
\text { reference to SRL } \\
\text { principles is a } \\
\text { good pedagogy } \\
\text { for enhancing } \\
\text { formative } \\
\text { learning } \\
\text { outcomes for } \\
\text { first-year US }\end{array}$ & $\begin{array}{l}1 \text { st year } \\
(n=73)\end{array}$ & SS & IT & OG-PP & $\begin{array}{l}\text { FC \& } \\
\text { SRL } \\
\text { principles } \\
\text { (two } \\
\text { classes: } \\
n 1=38, \\
n 2=36 \text { ) }\end{array}$ & - \\
\hline 14 & $\begin{array}{l}\text { Ozdamli } \\
\text { (2013) }\end{array}$ & TR & $\begin{array}{l}\text { Analyse } \\
\text { conditions } \\
\text { affecting } \\
\text { students' } \\
\text { perceptions of } \\
\text { self-directed } \\
\text { abilities \& } \\
\text { seamless } \\
\text { learning using } \\
\text { cloud systems \& } \\
\text { social network } \\
\text { applications }\end{array}$ & $\begin{array}{l}\text { US } \\
(n=40)\end{array}$ & SS & LM & QE-PP & $\begin{array}{l}\text { BL \& } \\
\text { MSSS \& } \\
\text { Evernote } \\
\& \text { social } \\
\text { network } \\
\text { applicatio } \\
\mathrm{n} \\
(n=40)\end{array}$ & - \\
\hline 15 & $\begin{array}{l}\text { Samruayruen } \\
\text { et al. (2013) }\end{array}$ & $\mathrm{TH}$ & $\begin{array}{l}\text { Examine } \\
\text { relationship } \\
\text { between } \\
\text { motivation \& } \\
\text { learning } \\
\text { strategies } \\
\text { sections \& } \\
\text { relationship }\end{array}$ & $\begin{array}{l}\text { UG/GD } \\
(n=88)\end{array}$ & $\mathrm{NM}$ & $\mathrm{NM}$ & OG-P & $\begin{array}{l}\text { BL \& } \\
\text { online } \\
\text { courses } \\
(n=88)\end{array}$ & - \\
\hline
\end{tabular}




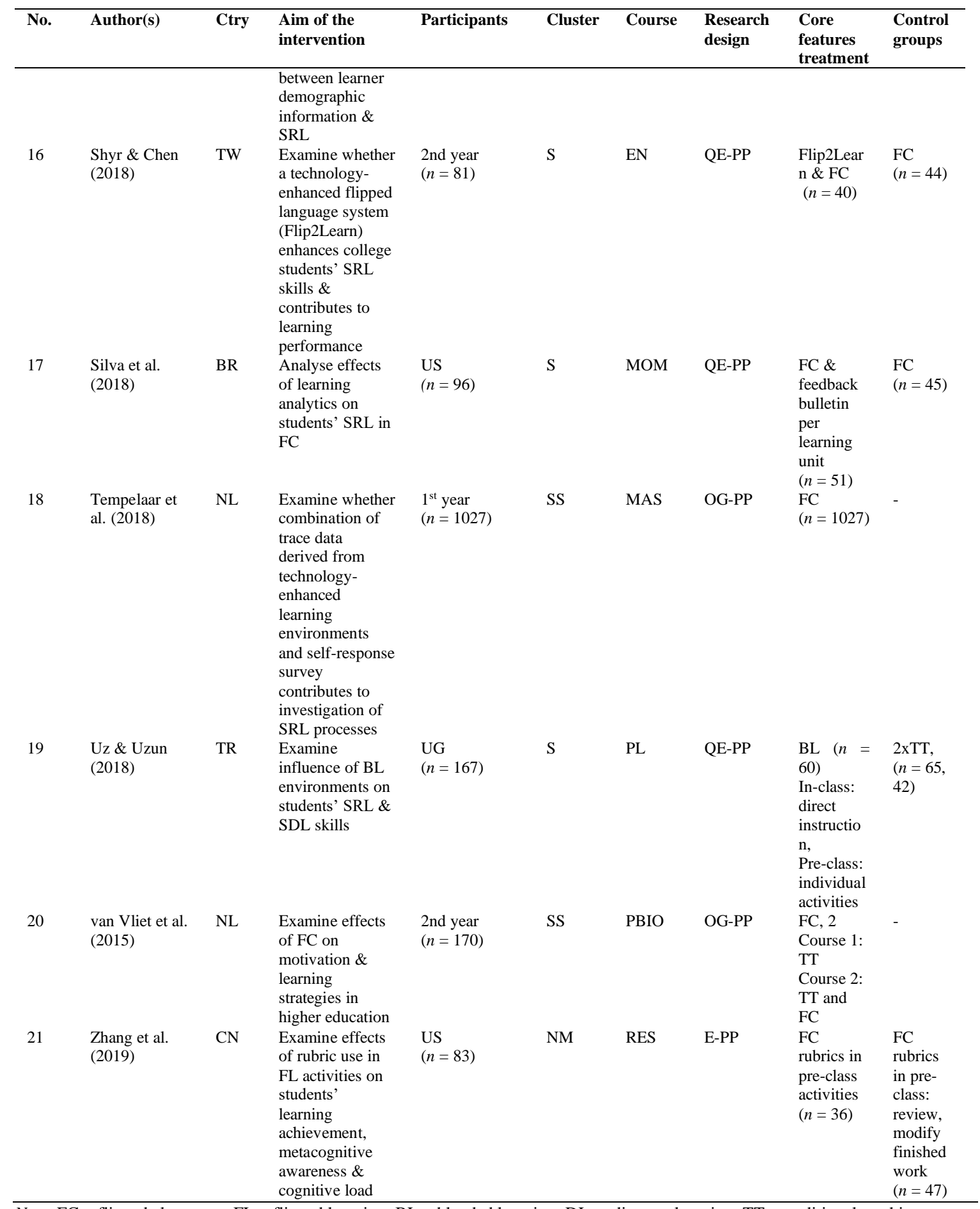

Note $. \mathrm{FC}=$ flipped classroom; FL = flipped learning; $\mathrm{BL}=$ blended learning; $\mathrm{DL}=$ distance learning; $\mathrm{TT}=$ traditional teaching; $\mathrm{NM}=$ not mentioned; - = not applicable.

Ctry: Country: $\mathrm{AU}=$ Australia; $\mathrm{BR}=$ Brazil; $\mathrm{CN}=\mathrm{China}$ IE = Ireland; NL = Netherlands; $\mathrm{ES}=\mathrm{Spain} ; \mathrm{SA}=\mathrm{Saudi}$ Arabia; $\mathrm{TH}=$ Thailand; TW = Thaiwan; TR = Turkey; USA = United States of America.

Participants: US = university students; $\mathrm{UG}=$ undergraduate; $\mathrm{GR}=$ graduate.

Cluster: $\mathrm{S}=$ Science; $\mathrm{SS}=$ Social science.

Course: RESM = Research Methods; BDSYS = Body System; MKT = Marketing; OS = Office Software; CMP = Computing; MOTF = Muscle of the Foot; LEUP = Legal \& Ethical Use of Property; NE = Nursing Ethics; JCM = Junior Cycle Medical; MI = Medical Immunology; PSY = Psychology; IT = Information Technology; LM = Learning Methods; EN = English; MOM = Mechanics of Materials; MAS = Mathematics \& Statistics; PL = Programming Languages; PBIO = Psycho Biology; RES = Research.

Research design: QE-PP = quasi-experiment pre-postttest; OG-P = one-group post only; OG-PP = one-group pre-posttest; E-P= experiment posttest only; E-PP = experiment pre-posttest.

Core features treatment: MSSLS = mobile supported seamless learning space; $A R=$ augmented reality 


\section{SRL strategies and interactional method}

Table 4 shows which SRL strategies were addressed in each study and which specific interactional methods were incorporated to promote SRL.

Table 4

Overview of SRL strategies per study and applied interactional methods, effect sizes broken down per strategy

\begin{tabular}{|c|c|c|c|c|c|c|c|c|}
\hline \multirow[t]{2}{*}{ Study } & \multirow[t]{2}{*}{ Strategy - subcategory } & \multicolumn{6}{|c|}{ Interactional methods } & \multirow{2}{*}{$\begin{array}{l}\text { Effects } \\
\text { size } \\
\text { reported }\end{array}$} \\
\hline & & H-ST & $\mathrm{H}-\mathrm{SS}$ & H-SE & NH-ST & NH-SC & NH-SE & \\
\hline \multirow[t]{7}{*}{1} & Metacognitive & $\mathrm{X}$ & & & & $\mathrm{X}$ & & - \\
\hline & Metacognitive - Monitoring & $X$ & & & & $X$ & & - \\
\hline & Motivation & $X$ & & & & & $X$ & - \\
\hline & Motivation & $X^{*}$ & & & & & $\mathrm{X}^{*}$ & M \\
\hline & $\begin{array}{l}\text { Motivation - Goal } \\
\text { orientation }\end{array}$ & $\mathrm{X}^{*}$ & & & & & $\mathrm{X}^{*}$ & M \\
\hline & Motivation - Task value & $\mathrm{X}^{*}$ & & & & & $\mathrm{X}^{*}$ & S \\
\hline & Motivation - Self-efficacy & $X^{*}$ & & & & & $\mathrm{X}^{*}$ & M \\
\hline \multirow[t]{4}{*}{2} & Metacognitive & $X^{*}$ & & & & $\mathrm{X}^{*}$ & & - \\
\hline & Cognitive - Elaboration & & $\mathrm{X}^{*}$ & & & $\mathrm{X}^{*}$ & & - \\
\hline & Cognitive - Rehearsal & & $\mathrm{X}^{*}$ & & & $\mathrm{X}^{*}$ & & - \\
\hline & Motivation - Self-efficacy & & $\mathrm{X}^{*}$ & & $\mathrm{X}^{*}$ & & & - \\
\hline \multirow[t]{5}{*}{3} & $\begin{array}{l}\text { Management - } \\
\text { Environment }\end{array}$ & & & & $\mathrm{X}^{*}$ & & & $\mathrm{~L}$ \\
\hline & Cognitive & $\mathrm{X}^{*}$ & & & & $\mathrm{X}^{*}$ & & M \\
\hline & Metacognitive - Planning & & & & $X$ & & & - \\
\hline & Management - Peers & $X^{*}$ & $\mathrm{X}^{*}$ & & $\mathrm{X}^{*}$ & & & M \\
\hline & Metacognitive - Evaluation & & & & $\mathrm{X}$ & & & - \\
\hline \multirow[t]{2}{*}{4} & Motivation Self-efficacy & & & & $\mathrm{X}^{*}$ & & & - \\
\hline & SRL strategies & & & & $X^{*}$ & & & - \\
\hline 5 & SRL strategies & $X^{*}$ & $\mathrm{X}^{*}$ & & $\mathrm{X}^{*}$ & & & $\mathrm{~L}$ \\
\hline 6 & Metacognitive & & & & $\mathrm{X}^{*}$ & & & - \\
\hline 7 & Metacognitive & & & & $\mathrm{X}^{*}$ & & & S \\
\hline 8 & Metacognitive & & & & $\mathrm{X}^{*}$ & & & $\mathrm{~L}$ \\
\hline \multirow[t]{4}{*}{9} & SRL strategies & & & & $\mathrm{X}^{*}$ & & & $\mathrm{~L}$ \\
\hline & Cognitive & & & & $\mathrm{X}^{*}$ & & & $\mathrm{~L}$ \\
\hline & Metacognitive - Monitoring & & & & $\mathrm{X}^{*}$ & & & $\mathrm{~L}$ \\
\hline & Metacognitive - Evaluation & & & & $\mathrm{X}^{*}$ & & & $\mathrm{~L}$ \\
\hline \multirow[t]{5}{*}{10} & $\begin{array}{l}\text { Motivation - Goal } \\
\text { orientation }\end{array}$ & $X^{*}$ & & & & & & - \\
\hline & Motivation - Self-efficacy & $\mathrm{X}^{*}$ & & & & & & - \\
\hline & Cognitive & & & & $\mathrm{X}^{*}$ & & & - \\
\hline & Metacognition & & & & $\mathrm{X}^{*}$ & & & - \\
\hline & Management - Peers & & & & $\mathrm{X}^{*}$ & & & - \\
\hline \multirow[t]{6}{*}{11} & Motivation & & & & $\mathrm{X}^{*}$ & & & - \\
\hline & SRL strategies & $\mathrm{X}^{*}$ & & & & & & - \\
\hline & Management-Peers & $\mathrm{X}$ & & & & & & - \\
\hline & Cognitive & $X$ & & & $X$ & & & - \\
\hline & Cognitive - Rehearsal & $\mathrm{X}^{*}$ & & & $\mathrm{X}^{*}$ & & & - \\
\hline & Metacognitive & $\mathrm{X}^{*}$ & & & & & & - \\
\hline \multirow[t]{4}{*}{12} & SRL strategies & & & & & $X^{*}$ & & $\mathrm{~L}$ \\
\hline & Metacognitive - Planning & & & & & $X^{*}$ & & $\mathrm{~L}$ \\
\hline & Metacognitive - Monitoring & & & & & $\mathrm{X}^{*}$ & & $\mathrm{~L}$ \\
\hline & Cognitive & & & & & $\mathrm{X}^{*}$ & & $\mathrm{~L}$ \\
\hline \multirow[t]{3}{*}{13} & Motivation - Self-efficacy & & & & & $X^{*}$ & & - \\
\hline & Metacognitive - Evaluation & & & & & $\mathrm{X}^{*}$ & & - \\
\hline & Management - Peers & $\mathrm{X}^{*}$ & & & & & & - \\
\hline 14 & SRL strategies & $\mathrm{X}^{*}$ & & & $\mathrm{X}^{*}$ & & & - \\
\hline \multirow[t]{2}{*}{15} & $\begin{array}{l}\text { Motivation - Goal } \\
\text { orientation }\end{array}$ & & & & & & $X^{*}$ & M \\
\hline & Motivation - Self-efficacy & & & & & & $X^{*}$ & $\mathrm{~L}$ \\
\hline
\end{tabular}




\begin{tabular}{|c|c|c|c|c|c|c|c|c|}
\hline \multirow[t]{2}{*}{ Study } & \multirow[t]{2}{*}{ Strategy - subcategory } & \multicolumn{6}{|c|}{ Interactional methods } & \multirow{2}{*}{$\begin{array}{l}\text { Effects } \\
\text { size } \\
\text { reported }\end{array}$} \\
\hline & & $\mathrm{H}-\mathrm{ST}$ & $\mathrm{H}-\mathrm{SS}$ & $\mathrm{H}-\mathrm{SE}$ & NH-ST & NH-SC & NH-SE & \\
\hline & Metacognitive & & & & & & $\mathrm{X}^{*}$ & $\mathrm{~L}$ \\
\hline 16 & SRL strategies & $X^{*}$ & $\mathrm{X}^{*}$ & & & $\mathrm{X}^{*}$ & & - \\
\hline \multirow[t]{4}{*}{17} & Metacognitive - Planning & $\mathrm{X}$ & & & & & $\mathrm{X}$ & - \\
\hline & $\begin{array}{l}\text { Management - } \\
\text { Environment }\end{array}$ & $\mathrm{X}$ & & & & & $\mathrm{X}$ & - \\
\hline & Management - Peer & $\mathrm{X}^{*}$ & & & & & $\mathrm{X}^{*}$ & M \\
\hline & Metacognitive - Evaluation & $X^{*}$ & & & & & $\mathrm{X}^{*}$ & M \\
\hline \multirow[t]{3}{*}{18} & Metacognitive & & & & & $\mathrm{X}^{*}$ & & - \\
\hline & Cognitive & & & & & $X^{*}$ & & - \\
\hline & Metacognitive - Planning & & & & & $X^{*}$ & & - \\
\hline 19 & SRL strategies & & & & & & $\mathrm{X}^{*}$ & - \\
\hline \multirow[t]{3}{*}{20} & Cognitive & & & & & $X^{*}$ & & - \\
\hline & Motivation - Task value & & & & & $X^{*}$ & $\mathrm{X}^{*}$ & - \\
\hline & Management - Peers & & $\mathrm{X}^{*}$ & & & & & - \\
\hline 21 & Metacognition & & & & $\mathrm{X}^{*}$ & & & $\mathrm{~S}$ \\
\hline
\end{tabular}

Note. Cohen's $d$ effect size was (S)mall $(0.20<d<0.50)$; (M)oderate $(0.50<d<0.80)$ or (L)arge $(d \geq$ $0.80) ;$ - = No effect size reported; * Significant effect.

H-ST: human student-teacher; H-SS: human student-student; H-SE: human student-expert; NH-ST: nonhuman student tool; NH-SC: non-human student content; NH-SE: non-human student environment.

\section{SRL strategies in general}

Eight studies investigated SRL strategies in general $(4,5,9,11,12,14,16,19)$. All studies reported a significant effect, but only three studies $(5,9,12)$ reported large effect sizes. Four studies $(4,9,12,19)$ used non-human interactional methods. The non-human student tool featured an online community for questions, discussion, support, sharing and reminders to each other to submit homework (4) or a pedagogical agent that asked students planning, monitoring and evaluation questions (9). One study (19) used the student environment to increase sharing amongst student through the online environment. Study 12 used student content through prompts embedded in videos.

Three studies $(5,14,16)$ used a combination of non-human and human interactional methods to foster SRL strategies. The non-human interactional method contained student tools including combining online media with the courses on the platform to facilitate home learning activities (5) and a cloud application to share results with peers and friends (14). Also, student content was provided, for example, prompts from a database to plan, monitor, modify and assess learning tasks (16).

Human interactional methods were applied for repeating the theory and teacher feedback (student-teacher) and peer feedback (student-student) in Study 5; peer feedback on the task (student-student) in Study 14, and metacognitive prompts as means of peer feedback on the task (student-student) and teacher feedback on skills and knowledge (student-teacher) in Study 16. One study (11) promoted SRL strategies in the human student-teacher interactional method through an interactive discussion to stimulate the students to analyse the questions thoroughly.

\section{Cognitive strategies}

Eight studies investigated cognitive strategies $(2,3,9,10,11,12,18,20)$, of which two studies studied specific cognitive strategies: rehearsal $(2,11)$ and elaboration $(2)$. Two studies $(11,18)$ did not report a significant effect on (general) cognitive strategies. Only three studies reported a moderate (3) or large (9, 12) effect size.

Five studies $(9,10,12,18,20)$ used non-human interactional methods to promote cognitive strategies: an online pedagogical agent (9) that prompted metacognitive questions in lectures notes and videos (student tool); a discussion forum (10) where students can ask questions and where students and faculty staff can respond to each other to help clarify teaching materials (student tool); prompts (12) embedded in videos (student content); tutorial, quizzes and exams (18) (student content); watching videos and submitting questions (20) to identify gaps in learning (student content). 
Three studies $(2,3,11)$ used a combination of human and non-human interactional methods: online individual quizzes $(2,3)$ or questioning via an online discussion forum (11) (student tool) together with team quizzes in class (2) (student-student) and discussions and providing additional information $(3,11)$ (student-teacher).

\section{Metacognitive strategies}

Fifteen studies investigated metacognitive strategies $(1,2,3,6,7,8,9,10,11,12,13,15,17,18,21)$, of which seven $(1,3,9,12,13,17,18)$ showed specific metacognitive strategies: planning $(3,12,17,18)$, monitoring $(1,9,12)$ and evaluation $(3,9,13,17)$. In four studies, there was no significant effect on metacognitive strategies in general $(1,18)$, for planning $(3,17)$, evaluation $(3)$ and monitoring $(1)$. Of the nine studies that were significant, only seven reported effect sizes: small (7, 21 - general strategies), moderate (17 - evaluation) or large (9 - monitoring and evaluation, 12 - monitoring and planning).

Eleven studies $(3,6,7,8,9,10,12,13,15,18,21)$ used non-human interactional methods. Seven studies $(3,6,7,8,9,10,21)$ used student tools: an instant response systems tool for posting, answering, information seeking, discussion and collective answer (3); augmented reality software with instructor notes (6); a discussion forum where students' work is assessed with a rubric (7); an online chat room presenting ethical dilemmas to a group of students and students reflecting on them with solutions for the dilemmas (8); a pedagogical agent that asked metacognitive questions in the videos (9); a discussion forum where students and staff post questions and where students and faculty staff can respond to each other to help clarify teaching material (10); and through a rubric with assessment criteria on smartphones to revise their work $(21)$. Two studies $(13,18)$ provided video learning materials in the format of a tutorial, quiz or exam that students could study at their own pace (student content). One study embedded prompts in videos (12) (student content). Two studies $(15,18)$ used online and hybrid experience $(15)$ or course schedules $(21)$ (student environment).

Three studies $(1,2,17)$ used a combination of non-human and human interactional methods to foster metacognitive strategies. Non-human interactional methods were watching content videos and weekly quizzes (1) (student content); individual quizzes (2) (student content); collecting information from the learning analytics (17) (student environment).

Human student-teacher interactional methods in the classroom were used for question and answer activities and discussion (1) or for answering quizzes and case-based learning facilitated by the teacher (2) or a bulletin board with feedback (17). One study used the human student-teacher interactional method through interactive discussion and communication to stimulate the students to thoroughly analyse the questions (11).

\section{Motivation strategies}

Seven studies $(1,2,4,10,13,15,20)$ examined specific motivation strategies: self-efficacy $(1,2,4,10,13$, $15)$, task value $(1,20)$ and goal orientation $(1,10,15)$. In one study, no significant effect was found on general motivation strategies (1). Of the eight studies with significant effects, only two reported a small (1 - task value), moderate ( 1 - self-efficacy, goal orientation, 15 - goal orientation) or large $(15$ - selfefficacy) effect size.

Five studies $(4,11,13,15,20)$ used non-human interactional methods; an online tool where students could request support from others (4) (student tool); lessons that can be taken any time by means of an app, depending on their own learning conditions (11) (student tool); videos to gain a better understanding of the content (13) (student content); daily use of the Internet (15) (student environment); recorded web lectures (20) (student content).

Two studies $(1,2)$ used a combination of human and non-human interactional methods by presenting different types of materials, by addressing students with different characteristics (student environment), by applying active learning activities such as discussions with direct feedback (student-teacher), an online quiz (student content) or collaborative learning (student-student). 
One study (10) used a human teacher-student interactional method and found that the quality of the teacher promoted students' goal orientation and self-efficacy.

\section{Management strategies}

Six studies $(3,10,11,13,17,20)$ investigated peer management strategies, two of which also investigated environment strategies $(3,17)$. Two studies $(11,17)$ found no significant effect on peers $(11)$ and the environment (17). Four studies showed significant results, but only two of them reported a moderate $(3,17$ - peers) or large (3 - environment) effect size.

Three studies $(11,13,20)$ used human interactional methods: individual or joined assignments to edit a picture while the teacher encourages peer support (13) (student-teacher); interactive discussions and class communications (11) (student-teacher); or discussions between students (20) (student-student).

Two studies $(3,17)$ reported a combination of non-human and human interactional methods: through a bulletin report with feedback on SRL that encouraged the search for support from other students to clarify doubts about the results (17) (student-teacher) collected from learning analytics (student environment); an online interactive response system, where students answered questions individually using their mobile devices (3) (student tool) and then formed groups (student-student) to seek information and discuss the questions with the teachers (student-teacher).

One study (10) used a non-human student tool through a discussion forum.

\section{Measurement instruments for SRL}

In total, 26 measurements instruments were reported (Table 5). Almost one fourth $(n=6)$ of the reviewed studies used the Motivated Strategies for Learning Questionnaire (MSLQ) as measurement instrument, followed by the Online Self-regulated Learning Questionnaire (OSLQ) $(n=2)$, the Self-directed Learning Readiness Scale (SLDRS) $(n=2)$ and focus groups $(n=2)$. The remaining instruments $(n=14)$ consisted of a compilation of already established and self-developed instruments to assess the different components of SRL. Most of the instruments were questionnaires $(n=23)$ followed by interviews $(n=2)$, think aloud $(n=1)$ and rubric $(n=1)$. Thirteen studies focused on metacognitive strategies: 9 on cognitive strategies; 5 on management strategies and 2 on SRL in general. For 10 of the 26 measures, no information on reliability was reported. Among the measures for which reliability was reported, values varied between acceptable to excellent (Tavakol \& Dennick, 2011). Three studies $(3,8,20)$ reported information about validity, for example, Study 8 reported an exploratory and Study 3 a confirmatory factor analysis.

Table 5

Key characteristics of the measurement instruments, broken down by total number of items, number of measurement scales, SRL strategies measured, scale format and test reliabilities (Cronbach's $\alpha$ )

\begin{tabular}{|c|c|c|c|c|c|c|}
\hline No. & Instrument & $\begin{array}{l}\text { No. of } \\
\text { items }\end{array}$ & $\begin{array}{l}\text { No. of } \\
\text { scales }\end{array}$ & SRL strategy & Scale format & $\alpha$ \\
\hline \multirow[t]{2}{*}{1} & $\begin{array}{l}\text { Motivation and Learning } \\
\text { Strategies Scale (MLSS) }\end{array}$ & 26 & 6 & Motivation & 7-point LS & NM \\
\hline & SDLRS & NM & 3 & $\begin{array}{l}\text { Metacognitive } \\
\text { Motivation }\end{array}$ & 5-point LS & .86 \\
\hline 2 & MSLQ & 31 & NM & $\begin{array}{l}\text { Motivation } \\
\text { SRL }\end{array}$ & 7-point LS & NM \\
\hline 3 & OSLQ & 24 & 6 & $\begin{array}{l}\text { Cognitive } \\
\text { Metacognitive } \\
\text { Management }\end{array}$ & 7-point LS & $>.92$ \\
\hline 4 & $\begin{array}{l}\text { MSLQ } \\
\text { SLDRS }\end{array}$ & $\begin{array}{l}7 \\
58\end{array}$ & $\begin{array}{l}\mathrm{NM} \\
\mathrm{NM}\end{array}$ & $\begin{array}{l}\text { Motivation } \\
\text { SRL }\end{array}$ & $\begin{array}{l}\text { 5-point LS } \\
\text { 5-point LS }\end{array}$ & $\begin{array}{l}\text { NM } \\
\text { NM }\end{array}$ \\
\hline 5 & SRLSs scale (questionnaire) & 38 & 4 & $\begin{array}{l}\text { Metacognitive } \\
\text { Cognitive } \\
\text { Management }\end{array}$ & 3-point LS & $>.76$ \\
\hline 6 & Questionnaire & NM & NM & Metacognitive & NM & NM \\
\hline 7 & $\begin{array}{l}\text { Logic-based-critical through } \\
\text { discussion grading rubric }\end{array}$ & - & - & Metacognitive & - & - \\
\hline
\end{tabular}




\begin{tabular}{|c|c|c|c|c|c|c|}
\hline No. & Instrument & $\begin{array}{l}\text { No. of } \\
\text { items }\end{array}$ & $\begin{array}{l}\text { No. of } \\
\text { scales }\end{array}$ & SRL strategy & Scale format & $\boldsymbol{\alpha}$ \\
\hline 8 & $\begin{array}{l}\text { Metacognition Scale } \\
\text { (questionnaire) }\end{array}$ & 28 & NM & Metacognitive & 5-point LS & $>.73$ \\
\hline 9 & SRL scale (questionnaire) & 59 & 3 & $\begin{array}{l}\text { Cognitive } \\
\text { Metacognition }\end{array}$ & 10-point LS & $>.95$ \\
\hline 10 & MSLQ & 81 & 3 & $\begin{array}{l}\text { Motivation } \\
\text { Cognitive } \\
\text { Metacognitive } \\
\text { Management }\end{array}$ & 7-point LS & NM \\
\hline 11 & $\begin{array}{l}\text { Survey paper APP-FC } \\
\text { teaching (questionnaire) }\end{array}$ & NM & NM & SRL & 5-point LS & $\mathrm{NM}$ \\
\hline 12 & Think aloud (scheme) & - & - & $\begin{array}{l}\text { Metacognitive } \\
\text { Cognitive }\end{array}$ & - & $\mathrm{NM}$ \\
\hline 13 & Focus group (interview) & - & - & - & $\begin{array}{l}\text { Open-ended } \\
\text { questions }\end{array}$ & - \\
\hline 14 & $\begin{array}{l}\text { Self-Rating Scale of Self- } \\
\text { Directed Learning } \\
\text { (questionnaire) }\end{array}$ & 60 & NM & SRL & 5-point LS & $>.95$ \\
\hline 15 & MSLQ & 44 & $\mathrm{NM}$ & $\begin{array}{l}\text { Motivation } \\
\text { Cognitive } \\
\text { Metacognitive }\end{array}$ & 7-point LS & $>.86$ \\
\hline 16 & $\begin{array}{l}\text { Online Learning Readiness } \\
\text { Scale (questionnaire) }\end{array}$ & 18 & 6 & $\begin{array}{l}\text { Management } \\
\text { Motivation } \\
\text { SRL }\end{array}$ & 5-point LS & .87 \\
\hline & MSLQ & 12 & NM & NM & 5-point LS & .82 \\
\hline 17 & OSLQ & 24 & 4 & $\begin{array}{l}\text { Metacognitive } \\
\text { Management }\end{array}$ & 5-point LS & $>.60$ \\
\hline 18 & $\begin{array}{l}\text { Student Approaches to } \\
\text { Learning framework } \\
\text { (questionnaire) }\end{array}$ & NM & NM & $\begin{array}{l}\text { Cognitive } \\
\text { Metacognitive }\end{array}$ & NM & $\mathrm{NM}$ \\
\hline \multirow[t]{2}{*}{19} & $\begin{array}{l}\text { SDLRS } \\
\text { Self-Regulated Learning } \\
\text { Scale (questionnaire) }\end{array}$ & $\begin{array}{l}\mathrm{NM} \\
\mathrm{NM}\end{array}$ & $\begin{array}{l}\mathrm{NM} \\
\mathrm{NM}\end{array}$ & $\begin{array}{l}\text { SRL } \\
\text { SRL }\end{array}$ & $\begin{array}{l}\mathrm{NM} \\
\mathrm{NM}\end{array}$ & $\begin{array}{l}.84 \\
.95\end{array}$ \\
\hline & Focus group (interview) & - & NM & - & $\begin{array}{l}\text { Open-ended } \\
\text { questions }\end{array}$ & - \\
\hline 20 & MSLQ & $\mathrm{NM}$ & 15 & $\begin{array}{l}\text { Motivation } \\
\text { Cognitive } \\
\text { Metacognitive } \\
\text { Management }\end{array}$ & 7-point LS & NM \\
\hline 21 & $\begin{array}{l}\text { Questionnaire on students' } \\
\text { metacognitive awareness }\end{array}$ & 37 & 2 & $\begin{array}{l}\text { Cognitive } \\
\text { Metacognitive }\end{array}$ & NM & .97 \\
\hline
\end{tabular}

Note. LS = Likert scale; NM = not mentioned; - = not applicable; APP-FC = application flipped classroom.

Cronbach's $\alpha<0.5$ unacceptable; $0.5 \leq \alpha<0.6$ poor; $0.6 \leq \alpha<0.7$ acceptable; $0.7 \leq \alpha<0.9$ good; $\alpha\rangle$ 0.9 excellent.

\section{Discussion and conclusion}

The main objective of this study was to review intervention studies about the role of SRL strategies in BL environments in higher education by answering two research questions:

(1) Which interactional methods in BL environments are effective for teaching and/or using SRL?

(2) Which instruments have been used to assess and measure SR learning in BL contexts?

With respect to the first research question, we identified four types of SRL strategies were distinguished: cognitive, metacognitive, motivation and management strategies. Most studies focused on metacognitive strategies. Cognitive strategies and motivation strategies were hardly addressed, while management strategies only received attention only in a few studies. Remarkably, little attention was paid to motivation strategies although this is a critical determinant for educational performance (Lazowski \& Hulleman, 2016). 
Researchers and practitioners increasingly recognise the importance of motivation (Broadbent \& Poon, 2015). Therefore, future research should pay attention to fostering motivation as an SRL strategy.

The interactional methods used in the studies showed that non-human student tools were widely applied, followed by human student-teacher interaction. Many studies used only non-human student tools to stimulate metacognitive strategies. Student tools that proved to be effective were, for example, a discussion forum and embedded prompts in videos. The other non-human interactional methods, such as student environment and student content, were used less often and were not always effective.

The human interactional method student-teacher was mainly used through discussion, group learning and feedback and applied for metacognitive strategies, cognitive strategies and SRL strategies in general.

The student-teacher methods, for example, answering questions through discussion and bulletin boards, were not always effective. The other human interactional method student-student, for example, the use of group work or peer feedback, was used less frequently, but appeared effective.

Only eight studies used a combination of human and non-human interactional methods. This finding is surprising, given that Nicol and Macfarlane-Dick (2006) have advocated the implementation of selfregulation training both in face-to-face and online settings.

The second research question was addressed by reporting measurement scales for cognitive and metacognitive SRL strategies. Commonly used measures were self-reporting and a combination of existing and self-developed instruments. Overall, the reliability scores of the reported measures were acceptable to excellent. Considering the SRL strategies measured, the MSLQ appears to be the most comprehensive instrument in terms of scales, items and supported strategies. Most studies have adapted or modified the original MSLQ instrument to address certain aspects of self-regulation. This finding is in line with the literature that MSLQ is one of the most widely used tools for measuring SRL strategies (Roth et al., 2016).

\section{Limitations}

This review study is subject to limitations. First, in most studies, students' SRL was measured using selfreports, which made it impossible to assess the actual quality of students' SRL strategies. Secondly, many studies reported about a relatively small sample in a specific curriculum context. We must therefore be cautious in making general recommendations. Thirdly, studies generally focused on undergraduate students in social sciences or science. Consequently, effects cannot be generalised to other student populations or curriculum content. Fourth, the studies in cases with treatment and control groups were not always easy to interpret because sometimes variations of BL were compared with each other and sometimes with traditional learning. Therefore, making comparative conclusions is difficult. A final limitation concerns the methodology. The studies were mainly quasi-experimental. Random assignment of participants to groups could not take place in most cases. Future experimental research is needed to replicate results and obtain more robust outcome measures.

\section{Implications}

This paper presents a classification of SRL strategies. By combining this classification with different human and non-human interactional methods, we analysed how self-regulation in BL environments was implemented in intervention studies in higher education. The results of this review suggest that SRL strategies can be successfully promoted through different interactional methods. Further research should provide more guidance for finding an optimal balance between human and non-human interactional methods to promote self-regulation strategies in different BL contexts in higher education.

The results show that cognitive and motivational strategies received little attention in BL environments, although these strategies are important for promoting self-regulation (Pintrich, 1990, 2004; Taub et al., 2014). With the increase of BL in higher education, more research in interactional methods that promote students' SRL is needed. In this regard, the student-student interactional method proved to be effective for collaboration and cooperative learning. According to Bernard et al. (2014), collaboration and cooperative learning not only promote learning but also strengthen elements of motivation and self-regulation strategies. The student tools interactional method proved to be promising for the promotion of metacognitive 
strategies. Further exploration of how student tools can be used to strengthen metacognitive and other selfregulation strategies is advised.

The framework presented in this evaluation uses key self-regulation strategies and (human and non-human) interactional methods. This framework can help lecturers and course designers to design new BL environments, communicate and share BL designs and evaluate existing BL practices.

Finally, the studies in our dataset mainly used the MSLQ to measure students' SRL. Our research has shown that the MSLQ only targets a subset of SRL strategies and therefore does not provide insight into the dynamic process of SRL. Therefore, we advocate the need for research on assessment instruments that aim to monitor the development of students' SRL strategy from the broader scope of the four SRL strategies used in our theoretical framework.

In the context of the COVID-19 pandemic, online and BL have become increasingly important. This review study emphasises the importance of research on SRL in BL environments. Despite the fact that attention to SRL is essential for students (cf. Barnard et al., 2009), this review shows that research on SRL in BL environments is scarce and offers few practical tools. The demand for practical tools to implement BL will increase rather than decrease in the near future; it is therefore important to conduct more research on how to effectively organise SRL in BL environments.

\section{References}

References with asterisk are included in this review.

Allen, I., Seaman, J., \& Garrett, R. (2007). Blending in: The extent and promise of blended education in the United States. Sloan-C. https://www.bayviewanalytics.com/reports/blending-in.pdf

*Alsancak Sirakaya, D., \& Ozdemir, S. (2018). The effect of a flipped classroom model on academic achievement, self-directed learning readiness, motivation and retention. Malaysian Online Journal of Educational Technology, 6(1), 76-91. http://www.ojsmojet.net/index.php/mojet/issue/view/21

*Atwa, S., Gauci-Mansour, V., Thomson, R., \& Hegazi, I. (2018). Team-based and case-based learning: A hybrid pedagogy model enhancing students' academic performance and experiences at first-year tertiary level. Australian Educational Researcher, 46(1), 93-112. https://doi.org/10.1007/s13384-018-0282-y

Azevedo, R., Cromley, J. G., \& Seibert, D. (2004). Does adaptive scaffolding facilitate students' ability to regulate their learning with hypermedia? Contemporary Educational Psychology, 29(3), 344-370. https://doi.org/10.1016/j.cedpsych.2003.09.002

Barnard, L., Lan, W. Y., To, Y. M., Paton, V. O., \& Lai, S. (2009). Measuring self-regulation in online and blended learning environments. The Internet and Higher Education, 12(1), 1-6. https://doi.org/10.1016/j.iheduc.2008.10.005

Bernard, R. M., Borokhovski, E., \& Schmid, R.F. (2014). A meta-analysis of blended learning and technology use in higher education: from the general to the applied. Journal of Computing in Higher Education. 26(1), 87-122. https://doi.org/10.1007/s12528-013-9077-3

Bersin \& Associates. (2003). Blended learning: What works? An industry study of the strategy, implementation, and impact of blended learning.

Bliuc, A., Goodyear, P., \& Ellis, R. A. (2007). Research focus and methodological choices in studies into students' experiences of blended learning in higher education. The Internet and Higher Education, 10(4), 231-244. https://doi.org/10.1016/j.iheduc.2007.08.001

Boekaerts, M. (1997). Self-regulated learning: A new concept embraced by researchers, policy makers, educators, teachers, and students. Learning and Instruction, 7(2), 161-186. https://doi.org/10.1016/S0959-4752(96)00015-1

Bonk, C. J., \& Graham, C. R. (2012). The handbook of blended learning: Global perspectives, local designs. John Wiley \& Sons.

Broadbent, J., \& Poon, W. L. (2015). Self-regulated learning strategies \& academic achievement in online higher education learning environments: A systematic review. The Internet and Higher Education, 27, 1-13. https://doi.org/10.1016/j.iheduc.2015.04.007

*Chen, P., \& Hwang, G. (2018). An IRS-facilitated collective issue-quest approach to enhancing students' learning achievement, self-regulation and collective efficacy in flipped classrooms. British Journal of Educational Technology, 50(4), 1996-2013. https://doi.org/10.1111/bjet.12690 
*Chyr, W. L., Shen, P. D., Chiang, Y. C., Lin, J. B., \& Tsai, C. W. (2017). Exploring the effects of online academic help-seeking and flipped learning on improving students' learning. Journal of Educational Technology \& Society, 20(3), 11-23. https://www.j-ets.net/collection/published-issues/20_3

de Bruijn-Smolders, M., Timmers, C. F., Gawke, J. C., Schoonman, W., \& Born, M. P. (2016). Effective self-regulatory processes in higher education: Research findings and future directions. A systematic review. Studies in Higher Education, 41(1), 139-158. https://doi.org/10.1080/03075079.2014.915302

Dignath, C., Buettner, G., \& Langfeldt, H. (2008). How can primary school students learn self-regulated learning strategies most effectively? Educational Research Review, 3(2), 101-129. https://doi.org/10.1007/s10648-008-9083-6

Dinsmore, D. L., Alexander, P. A., \& Loughlin, S. M. (2008). Focusing the conceptual lens on metacognition, self-regulation, and self-regulated learning. Educational Psychology Review, 20(4), 391-409. https://doi.org/10.1007/s10648-008-9083-6

Driscoll, M. (2002). Blended learning: Let's get beyond the hype. (The last word). E-Learning, 1(4), 1-4.

Duncan, T., \& McKeachie, W. (2005). The making of the motivated strategies for learning questionnaire. Educational Psychologist, 40(2), 117-128. https://doi.org/10.1207/s15326985ep4002_6

Dziuban, C., Graham, C., \& Picciano, A. G. (2013). Research perspectives in blended learning (2nd ed.). Routledge.

*El-Senousy, H., \& Alquda, J. (2017). The effect of flipped classroom strategy using blackboard mash-up tools in enhancing achievement and self-regulated learning skills of university students. World Journal on Educational Technology: Current Issues, 9(3), 144-157. https://doi.org/10.18844/wjet.v6i3.1974

*Ferrer-Torregrosa, J., Jiménez-Rodríguez, M., Torralba-Estelles, J., Garzón-Farinós, F., Pérez-Bermejo, M., \& Fernández-Ehrling, N. (2016). Distance learning ects and flipped classroom in the anatomy learning: Comparative study of the use of augmented reality, video and notes. BMC Medical Education, 16(1), 1-9. https://doi.org/10.1186/s12909-016-0757-3

*Giacumo, L., \& Savenye, W. (2019). Asynchronous discussion forum design to support cognition: Effects of rubrics and instructor prompts on learner's critical thinking, achievement, and satisfaction. Educational Technology Research and Development, 68(1), 37-66. https://doi.org/10.1007/s11423019-09664-5

Graham, C. R. (2006). Blended learning systems: Definition, current trends, and future directions. In C. J. Bonk \& C. R. Graham (Eds.), Handbook of blended learning: Global perspectives, local designs (pp. 3-21). Pfeiffer Publishing.

Hanna, D. E., Glowacki-Dudka, M., \& Conceiçáo-Runlee, S. (2000). 147 Practical tips for teaching online groups: Essentials of web-based education. Atwood Publishing. https://doi.org/10.1016/S1096$\underline{7516(02) 00108-2}$

Harackiewicz, J., Barron, K., Pintrich, P., Elliot, A., \& Thrash, T. (2002). Revision of achievement goal theory: Necessary and illuminating. Journal of Educational Psychology, 94(3), 638-645. https://doi.org/10.1037/0022-0663.94.3.638

Harasim, L. (2000). Shift happens online education as a new paradigm in learning. The Internet and Higher Education, 3(1), 41-61. https://doi.org/10.1016/S1096-7516(00)00032-4

Hattie, J., Biggs, J., \& Purdie, N. (1996). Effects of learning skills interventions on student learning: A meta-analysis. Review of Educational Research, 66(2), 99-136. https://doi.org/10.3102/00346543066002099

*Hsu, L., \& Hsieh, S. (2014). Factors affecting metacognition of undergraduate nursing students in a blended learning environment. International Journal of Nursing Practice, 20(3), 233-241. https://doi.org/10.1111/ijn.12131

*Karaoğlan Yılmaz, F. G., Olpak, Y. Z., \& Y1lmaz, R. (2018). The effect of the metacognitive support via pedagogical agent on self-regulation skills. Journal of Educational Computing Research, 56(2), 159189. https://doi.org/10.1177/0735633117707696

*Kassab, S. E., Al-Shafei, A., Salem, A., \& Otoom, S. (2015). Relationships between the quality of blended learning experience, self-regulated learning, and academic achievement of medical students: A path analysis. Advances in Medical Education and Practice, 6, 273-4. https://doi.org/10.2147/AMEP.S75830

Lazowski, R. A., \& Hulleman, C. S. (2016). Motivation interventions in education: A meta-analytic review. Review of Educational Research, 86(2), 602-640. https://doi.org/10.3102/0034654315617832

Loyens, S., Magda, J., \& Rikers, R. (2008). Self-directed learning in problem-based learning and its relationships with self-regulated learning. Educational Psychology Review, 20(4), 411-427. https://doi.org/10.1007/s10648-008-9082-7 
*Ma, X., Luo, Y., Zhang, L., Wang, J., Liang, Y., Yu, H., Wu, Y., Tan, Y., \& Cao, M. (2018). A trial and perceptions assessment of APP-based flipped classroom teaching model for medical students in learning immunology in China. Education Sciences, 8(2). https://doi.org/10.3390/educsci8020045

Mayer, R. E. (2008). Learning and instruction (2nd ed). Pearson Merrill Prentice Hall.

Moher, D., Liberati, A., Tetzlaff, J., \& Altman, D., \& Prisma Group. (2009). Preferred reporting items for systematic reviews and meta-analyses: The PRISMA statement. Annals of Internal Medicine, 151(4), 264-269. https://doi.org/10.7326/0003-4819-151-4-200908180-00135

*Moos, D. C., \& Bonde, C. (2016). Flipping the classroom: Embedding self-regulated learning prompts in videos. Technology, Knowledge and Learning, 21(2), 225-242. https://doi.org/10.1007/s10758015-9269-1

*Ng, E. M. W. (2018). Integrating self-regulation principles with flipped classroom pedagogy for first year university students. Computers and Education, 126(1), 65-74. https://doi.org/10.1016/j.compedu.2018.07.002

Nicol, D., \& Macfarlane-Dick, D. (2006). Formative assessment and self-regulated learning: A model and seven principles of good feedback practice. Studies in Higher Education, 31(2), 199-218. https://doi.org/10.1080/03075070600572090

*Ozdamli, F. (2013). Effectiveness of cloud systems and social networks in improving self-directed learning abilities and developing positive seamless learning perceptions. Journal of Universal Computer Science, 19(5), 602-619. https://doi.org/10.3217/jucs-019-05-0602

Pintrich, P. (2000). The role of goal orientation in self-regulated learning. In M. Boekaerts, P. Pintrich, \& M. Zeidner (Eds.), Handbook of self-regulation (pp. 451-502). Academic Press. https://doi.org/10.1016/B978-012109890-2/50043-3

Pintrich, P. (2003). A motivational science perspective on the role of student motivation in learning and teaching contexts. Journal of Educational Psychology, 95(4), 667-686. https://doi.org/10.1037/00220663.95 .4 .667

Pintrich, P. (2004). A conceptual framework for assessing motivation and self-regulated learning in college students. Educational Psychology Review, 16(4), 385-407. http://doi.org/10.1007/s10648004-0006-x

Pintrich, P., Smith, D., Garcia, T., \& McKeachie, W. (1991). A manual for the use of the Motivated Strategies For Learning Questionnaire (Technical Report 91-B-004). The Regents of the University of Michigan (ED338122). ERIC. https://eric.ed.gov/?id=ED338122

Roth, A., Ogrin, S., \& Schmitz, B. (2016). Assessing self-regulated learning in higher education: A systematic literature review of self-report instruments. Educational Assessment, Evaluation and Accountability, 28(3), 225-250. https://doi.org/10.1007/s11092-015-9229-2

*Samruayruen, B., Enriquez, J., Natakuatoong, O., \& Samruayruen, K. (2013). Self-regulated learning: A key of a successful learner in online learning environments in Thailand. Journal of Educational Computing Research, 48(1), 45-69. https://doi.org/10.2190/EC.48.1.c

Schraw, G., \& Dennison, R. (1994). Assessing metacognitive awareness. Contemporary Educational Psychology, 19(4), 460-475. https://doi.org/10.1006/ceps.1994.1033

Sharma, S., Dick, G., Chin, W., \& Land, L. (2007). Self-regulation and e-learning. In H. Osterle, J. Schelp \& R. Winter (Eds.), Proceedings of the Fifteenth European Conference on Information Systems (pp. 383-394). Association for Information Systems. https://aisel.aisnet.org/ecis2007/45

*Shyr, W., \& Chen, C. (2018). Designing a technology-enhanced flipped learning system to facilitate students' self-regulation and performance. Journal of Computer Assisted Learning, 34(1), 53-62. https://doi.org/10.1111/jcal.12213

*Silva, J. C. S., Zambom, E., Rodrigues, R. L., Ramos, J. L. C., \& de Souza, F. D. F. (2018). Effects of learning analytics on students' self-regulated learning in flipped classroom. International Journal of Information and Communication Technology Education, 14(3), 91-107. https://doi.org/10.4018/IJICTE.2018070108

Sitzmann, T., \& Ely, K. (2011). A meta-analysis of self-regulated learning in work-related training and educational attainment: What we know and where we need to go. Psychological Bulletin, 137(3), 421-442. https://doi.org/10.1037/a0022777

Spanjers, I., Könings, K., Leppink, J., Verstegen, D., de Jong, N., Czabanowska, K., \& van Merriënboer, J. (2015). The promised land of blended learning: Quizzes as a moderator. Educational Research Review, 15, 59-74. https://doi.org/10.1016/j.edurev.2015.05.001

Staker, H., \& Horn, M. B. (2012). Classifying K-12 blended learning. Innosight Institute.

Taub, M., Azevedo, R., Bouchet, F., \& Khosravifar, B. (2014). Can the use of cognitive and metacognitive self-regulated learning strategies be predicted by learners' levels of prior knowledge in 
hypermedia-learning environments? Computers in Human Behavior, 39, 356-367. https://doi.org/10.1016/j.chb.2014.07.018

Tavakol, M., \& Dennick, R. (2011). Making sense of Cronbach's alpha. International Journal of Medical Education, 2, 53-55. https://doi.org/10.5116/ijme.4dfb.8dfd

*Tempelaar, D., Rienties, B., \& Nguyen, Q. (2018). A multi-modal study into students' timing and learning regulation: Time is ticking. Interactive Technology and Smart Education. 15(4), 298-313. https://doi.org/10.1108/ITSE-02-2018-0015

*Uz, R., \& Uzun, A. (2018). The influence of blended learning environment on self-regulated and selfdirected learning skills of learners. European Journal of Educational Research, 7(4), 877-886. https://doi.org/10.12973/eu-jer.7.4.877

*van Vliet, E. A., Winnips, J. C., \& Brouwer, N. (2015). Flipped-class pedagogy enhances student metacognition and collaborative-learning strategies in higher education but effect does not persist. CBE - Life Sciences Education, 14(3), 1-10. https://doi.org/10.1187/cbe.14-09-0141

Ward, J., \& LaBranche, G. A. (2003). Blended learning: The convergence of e-learning and meetings. Franchising World, 35(4), 22-24.

Weinstein, C. E., Husman, J., Dierking, D. R. (2000). Self-regulation interventions with a focus on learning strategies. Handbook of self-regulation (pp. 727-747). Academic Press. https://doi.org/10.1016/B978-012109890-2/50051-2

*Zhang, Y., Chen, B. L., Ge, J., Hung, C. Y., \& Mei, L. (2019). When is the best time to use rubrics in flipped learning? A study on students' learning achievement, metacognitive awareness, and cognitive load. Interactive Learning Environments, 27(8), 1207-1221. https://doi.org/10.1080/10494820.2018.1553187

Zheng, L. (2016). The effectiveness of self-regulated learning scaffolds on academic performance in computer-based learning environments: A meta-analysis. Asia Pacific Education Review, 17(2), 187202. https://doi.org/10.1007/s12564-016-9426-9

Corresponding author: Jan Hein Eggers, j.h.eggers@ @ hva.nl

Copyright: Articles published in the Australasian Journal of Educational Technology (AJET) are available under Creative Commons Attribution Non-Commercial No Derivatives Licence (CC BY$N C-N D$ 4.0). Authors retain copyright in their work and grant AJET right of first publication under CC BY-NC-ND 4.0.

Please cite as: Eggers. J. H., Oostdam, R., \& Voogt, J. (2021). Self-regulation strategies in blended learning environments in higher education: A systematic review. Australasian Journal of Educational Technology, 37(6), 175-192. https://doi.org/10.14742/ajet.6453 